\title{
Diseño Universal del Aprendizaje e inclusión en la Educación Básica
}

\section{Universal Design of Learning and and inclusion in Basic Education}

Dra. Rosa M. Espada Chavarría es docente e Investigadora de la Universidad Rey Juan Carlos (España) (rosa.espada@urjc.es) (D) (https://orcid.org/0000-000 1-8386-3298)

Dra. Miriam Bernarda Gallego Condoy es docente e Investigadora de la Universidad Politécnica Salesiana (Ecuador) (mgallego@ups.edu.ec) (D) (https://orcid.org/0000-0003-4684-5984)

Dr. Rayco H. González-Montesino es docente e Investigador de la Universidad Rey Juan Carlos (España) (raycoh.gonzalez@urjc.es) (D) (https://orcid.org/0000-0002-6830-3951)

Recibido: 2019-01-30 / Revisado: 2019-04-23 / Aceptado: 2019-05-16 / Publicado: 2019-07-01

\section{Resumen}

La educación inclusiva se concreta en las aulas atendiendo a la diversidad del alumnado, lo que supone el paso necesario para garantizar la igualdad de oportunidades en el acceso al proceso educativo. E presente trabajo pretende conocer las metodologías y estrategias didácticas que utilizan los docentes de educación básica y si estas se basan en el diseño universal para el aprendizaje (DUA). La metodología es de corte cuantitativo de alcance descriptivo, para el que se utilizó un cuestionario de escala tipo Likert de 29 ítems. Como complemento al estudio se realizan, además, 4 preguntas abiertas de carácter cualitativo. La muestra final es de 26 docentes, que trabajan con niños entre 7 y 12 años, de cuatro establecimientos educativos representando a tres de las regiones naturales del Ecuador. Los resultados principales reflejan un conocimiento limitado del DUA y sus principios, destacando que en las escuelas publicas y en aquellas que existe un porcentaje mas alto de estudiantes con discapacidad, solo el 29\% de los docentes conocen de manera aproximada lo que significa el DUA. Por otro lado, se observan actuaciones que afectan al proceso formativo y a la igualdad de oportunidades de estudiantes que presentan baja audición y visión. Concluimos con la necesidad de capacitación docente en DUA como alternativa didáctica en el marco de la educación inclusiva al proporcionar las mismas oportunidades a todo el estudiantado independientemente de sus características individuales.

Descriptores: Diseño Universal para el Aprendizaje, inclusión educativa, igualdad de oportunidades, accesibilidad, diversidad, estrategias didácticas

\begin{abstract}
Inclusive education is made concrete in the classroom, taking into account the diversity of pupils, which is the necessary step to ensure equal opportunities in access to the educational process. The objective of this paper is to know the methodologies and didactic strategies used by basic education teachers and whether these are based on the Universal Design for Learning (UDL). The methodology is quantitative and descriptive in scope, using a Likert scale question-
\end{abstract}

Forma sugerida de citar: Espada Chavarría, R. M., Gallego Condoy, M., \& González-Montesino, R.H. (2019). Diseño Universal del Aprendizaje e inclusión en la Educación Básica. Alteridad, 14(2), 207-218. https://doi.org/10.17163/alt.v14n2.2019.05 
naire with 29 items. As a complement to the study, four qualitative open-ended questions are also carried out. The final sample is 26 teachers, who work with children between 7 and 12 years old, from four educational establishments representing three of the natural regions of Ecuador. The main results reflect a limited knowledge of the UDL and its principles, highlighting that in public schools and in those where there is a higher percentage of students with disabilities, only $29 \%$ of teachers know approximately what the UDL

\section{Introducción y estado de la cuestión}

La educación inclusiva es considerada como un medio eficaz para educar a todos, independientemente de sus diferencias o barreras individuales o sociales (UNESCO, 2015). Sin embargo, esta, requiere de estrategias para llevar acabo sus principios de igualdad de oportunidades, atención y respecto a la diversidad, accesibilidad, entre otras (Blanco, \& Duck, 2011). El diseño universal del aprendizaje (DUA) aparece como una respuesta a la viabilidad de los principios de la educación inclusiva (Sánchez, Díez, \& Martín, 2016), comprendiéndose así como una estrategia didáctica que aplica los principios universales del diseño universal al diseño del currículo, de tal modo que, el aprendizaje pueda llegar a todos los alumnos de una manera equitativa, en donde los materiales didácticos utilizados a través de los medios tecnológicos, renueven la utilización de los materiales tradicionales rígidos, carentes de creatividad, poco funcionales y creativos; $y$, con esto se de atención a la diversidad del alumnado y las diferentes habilidades sensoriales, motrices, cognitivas, afectivas y lingüísticas (Díez, \& Sánchez, 2015).

En el contexto ecuatoriano, la Ley Orgánica de Educación e Interculturalidad (LOEI) en los artículos 11 y 12, manifiesta que: la educación inclusiva es el proceso de identificar y responder a la diversidad de necesidades de los estudiantes a través de la mayor participación en el aprendizaje de las culturas, de las comunidades y de las personas que se encuentran excluidas del sistema means. On the other hand, there are actions that affect the formative process and the equality of opportunities for students with low hearing and vision. We conclude with the need for teacher training in UDL as a didactic alternative in the framework of inclusive education by providing the same opportunities to all students regardless of their individual characteristics.

Keywords: Universal Design for Learning, inclusion, equal opportunities, accessibility, diversity, teaching strategies.

educativo, a fin de reducir la exclusión en la educación (Ministerio de Educación, 2011). En la educación inclusiva y en la aplicación de estrategias metodológicas del DUA, el rol docente juega un papel importante, puesto que el enfoque inclusivo, implica la implantación de recursos y estrategias que permitan a la comunidad educativa y concretamente al profesorado, afrontar con éxito los cambios que suponen esta nueva practica educativa (Díez, \& Sánchez, 2015).

Por lo que resulta importante conocer cuál es la comprensión de los docentes sobre el diseño universal del aprendizaje y, qué estrategias y didácticas metodológicas identificadas con tales principios, aplican en sus prácticas educativas. En la práctica docente, el profesorado realiza un conjunto de actividades, métodos y técnicas, así como utiliza medios de lenguajes, materiales y símbolos para representar conceptos y evaluación. Por lo tanto, es importante conocer en qué medida, los docentes atienden a la diversidad del alumnado y como garantizan la igualdad de oportunidades en el acceso, participación y desarrollo del currículo (Sánchez, Díez, \& Martín, 2016). Por otro lado, es necesario destacar papel relevante que jugará el aspecto emocional en el proceso de aprendizaje a través de estrategias que utilicen el DUA.

El componente emocional es un elemento crucial en el aprendizaje. En él se pone en evidencia las diferencias, por lo que es importante conocer las formas de atender a la diversidad desde el componente emocional, saber cómo el docente motiva a los estudiantes a aprender. La declaración de Salamanca, en su compromiso de conseguir una 
educación para todos, plantea que el acceso a la escuela debe de darse a todos los niños. Los centros educativos, deben acoger a niños y niñas con discapacidad, con altas capacidades, en situación de riesgo de exclusión social, independientemente de su etnia y/o cultura (Espada, 2017).

Por este motivo nuestra investigación centra su atención en la educación regular, en el nivel de educación general básica, puesto que es en los niveles de educación básica donde se debe garantizar una educación de calidad, la misma que influirá en la práctica de una cultura inclusiva. El Ecuador es un país pluricultural y multicultural.

Según el Artículo 1 de la Constitución vigente, el Ecuador es un país plurinacional y multicultural, además un país diverso, fortalecido por las semejanzas de su pueblo, con habitantes procedentes de raíces ancestrales diversas como: mestizos, indígenas, montubios y afro ecuatorianos. (Asamblea Constituyente, 2008) Por otro lado, entre Amazonía, Sierra y Costa, el país cuenta con 15 nacionalidades indígenas (Benítez, \& Garcés, 2016). Por lo que, la educación en este contexto está matizada por la diversidad de estudiantes de los diferentes grupos que forman parte de la nación, siendo las escuelas ordinarias el punto de encuentro y espacio clave que promocione una sociedad integradora y que evite actitudes y situaciones de discriminación, creando, además comunidades de acogida que logren una educación para todos. (UNESCO, 1994).

Es por ello importante conocer cómo se está haciendo realidad la educación para todos, mediante la práctica del diseño universal del aprendizaje en el nivel de Educación Básica. Es así como, el objetivo de la investigación es identificar tipo de conocimiento sobre diseño universal para el aprendizaje de los docentes de educación básica y analizar el nivel aplicación que realizan de éste en sus aulas. De este modo podremos valorar las prácticas educativas y detectar las necesidades en la formación de los docentes que garantice una educación de calidad, fomentando la inclusión educativa y el diseño universal para el aprendizaje.

El desconocimiento de dicha práctica puede estar comprometiendo la educación para todos, tal como lo plantea la declaración de Inchon, que establece como objetivo de desarrollo sostenible para el 2030 "garantizar una educación inclusiva y equitativa de calidad y promover oportunidades de aprendizaje para todas las personas a lo largo de toda la vida" (UNESCO, 2015).

El DUA, que inicialmente surge relacionado con la arquitectura, y posteriormente con la elaboración de productos y servicios, entra en el campo educativo apoyado de las herramientas tecnológicas, llegando a definirse en la actualidad como guía referencial de la educación para todos. Según Rose y Mayer creadores del DUA, se sirvieron de la tecnología para identificar tres redes diferenciadas que intervienen en los procesos de aprendizaje: "Red de reconocimiento (el 'qué' del aprendizaje), red estratégica (el 'cómo' del aprendizaje), y la red afectiva (la implicación en el aprendizaje)" (Alba, 2015). Es así como el DUA se fundamenta en cuatro argumentos que son: la neurociencia, el aprendizaje cognitivo, la tecnología de la información (TIC) y la educación.

En cuanto a la neurociencia, se indica que el cerebro del niño aprende más cuando el alumno explica a sus compañeros sobre un tema en particular y, aprende más, después que realiza una actividad física. Así, la actividad cerebral se puede caracterizar en función de tres redes cerebrales que participan en el aprendizaje, estas son: redes de reconocimiento, redes de estrategias y redes afectivas (Barrera, 2009). En las redes de reconocimiento, el niño percibe e identifica la información del exterior; hace referencia al "que del aprendizaje". En las redes de estrategias, el niño planifica, ejecuta tareas y expresa ideas de diversa forma; hace referencia al "como del aprendizaje. En las redes afectivas, el niño se motiva y se compromete con el aprendizaje; es el "porque del aprendizaje”. (Barrera, 2009).

De estas tres redes de relaciones surgen los tres principios fundamentales del diseño 
universal para el aprendizaje que son: principio múltiples formas de representación, principio múltiples formas de acción y expresión, y principio de múltiples formas de implicación; haciendo notar que el funcionamiento de cada una de estas subredes es distinto en cada persona (Alba, Sánchez, \& Zubillaga, 2013). El principio de múltiples formas de representación se centra en los diferentes modos en los que el alumnado percibe y comprende la información facilitada. De ahí, la importancia de facilitar variedad de opciones relacionadas con la información auditiva, visual o táctil, lo que supone proporcional múltiples opciones para el lenguaje y los símbolos (Alba, 2015), que faciliten la compresión mediante la activación de los conocimientos previos que se encuentran almacenados en el individuo, debido a sus experiencias pasadas (Ausubel, 2002).

El principio de múltiples formas de acción y expresión se basa en las diferentes maneras que el alumnado puede desenvolverse en un entorno de aprendizaje, ya que es posible que algunos alumnos tengan una mayor habilidad para la expresión escrita que en la expresión verbal y viceversa. Del mismo modo, pueden darse diferencias a la hora de ejecutar tareas, de ahí que sea necesario manejar opciones diversas en los procesos de expresión y acción. Respecto al principio de múltiples formas de implicación, sostiene que el componente afectivo activa una red cerebral que interviene en el aprendizaje.

De este modo, se establece la parte afectiva como pieza clave en el proceso de aprendizaje, lo que supone que cada estudiante presente una diferencia importante en la implicación o motivación por aprender o qué aprender, así como también muestran preferencia respecto tipo de colaboración en la ejecución de las actividades, prefiriendo en unos casos el trabajo individual y en otros el trabajo colectivo. Este hecho supone que el hecho de facilitar diferentes vías de participación sea preciso no solo para potenciar la motivación e implicación, sino también para permitir un equilibrio emocional.

\section{Material y métodos}

Se realiza un tipo de investigación descriptiva. Teniendo en cuenta que los cuestionarios son instrumentos de investigación que permiten obtener información sistemática y ordenada sobre un amplio abanico de cuestiones a la vez, hemos hecho uso de ellos para recopilar la máxima información posible sobre los indicadores y preguntas propuestas por el Diseño Universal del Aprendizaje (DUA). Por todo ello, se ha realizado un modelo de cuestionario de escala tipo Likert de 29 ítems que incorpora preguntas relacionadas con los tres principios del DUA: representación de la información, acción y expresión, motivación e implicación del aprendizaje.

El instrumento se basa en los aportes del CAST (2011) (Center for Applied Special Technology) entidad investigadora que centra sus esfuerzos en fomentar, promover y expandir el diseño universal del aprendizaje, promoviendo así las oportunidades de aprendizaje para todos, y centrándose en aquellas personas que presentan algún tipo de discapacidad. Se ha utilizado la versión de 2013 traducida por Alba, Sánchez, \& Zubillaga (2013).

La información se complementa con cuatro preguntas abiertas de carácter cualitativo, orientadas al conocimiento del DUA, al significado de la educación y cultura inclusiva, a las estrategias docentes utilizadas para favorecer inclusión educativa junto con la valoración de la necesidad de formación docente en estrategias de diseño universal para el aprendizaje, por lo que podemos considerar la metodología utilizada como de tipo mixto, ya que no solo presenta datos cuantitativos al utilizarse cuestionarios como instrumento de recogida de datos, sino que también incorpora elementos de la metodología cualitativa al incluir literalmente la información de los participantes, ya sean estas habladas o escritas (Quecedo, \& Castaño, 2003).

La muestra inicial es de 28 docentes, siete por centro educativo, siendo el criterio de inclusión que tengan a su cargo niños de educación básica elemental y media, es decir, docentes de 
trabajen con niños con edades comprendidas entre 7 y 12 años. Finalmente, no respondieron 2 docentes de la escuela fiscomisional, quedando la muestra en 26 docentes ( 9 varones y 17 mujeres), con una edad comprendida entre los 26 a 60 años. Para seleccionar los establecimientos educativos se ha atendido a los siguientes criterios: tipo de establecimiento reglamentado por el Ministerio de Educación (pública o fiscal, fiscomisional, privada o particular y bilingüe intercultural) y las regiones naturales de Ecuador, identificándose uno de Costa, dos de Sierra y uno en la región Amazónica, no habiendo sido posible contar con una referencia de la región insular.

De este modo, contamos con un centro particular de la ciudad de Quito, provincia de Pichincha (7 docentes); un establecimiento fiscal ubicado en el Catón la Concordia a $200 \mathrm{~km}$ de la capital, con cercanía a las ciudades de Santo Domingo y Esmeraldas (7 docentes); un establecimiento fiscomisional en la Provincia de Loja ubicado a $860 \mathrm{~km}$ de la capital (línea fronteriza entre Ecuador y Perú) (5 docentes); y un establecimiento educativo intercultural bilingüe ubicado en la provincia de Sucumbíos, a $300 \mathrm{~km}$ de la capital y a $30 \mathrm{~km}$ de la frontera con Colombia (7 docentes). Las personas encuestadas son en su mayoría mujeres y el rango de edad oscila de 24 a 35 años, llamando la atención los docentes de la institución fiscomisional de Loja que cuentan con un rango de edad comprendido entre 46 y 60 años. En cuanto al nivel formativo, 25 profesores cuentan con título de tercer nivel y un docente con título de cuarto nivel.

\section{Análisis y resultados}

A la vista de los resultados obtenidos, destacamos las observaciones que revelan mayor aporte para su análisis, ya sea por su mayor frecuencia o por considerarse más relevantes para entender la práctica del DUA. Tal y como refleja la tabla 1 , se percibe que la presencia de estudiantes con discapacidad se da más en los establecimientos fiscales (Santo Domingo) y fiscomisionales (Loja) antes que en un establecimiento particular (Pichincha) o intercultural bilingüe (Sucumbíos).

Sin embargo y atendiendo a los datos mostrados en la tabla 2, pese a que la LOEI establece en sus principios el derecho a la educación como bien de todos y el Ministerio de Educación desde diferentes ámbitos ofrece espacios de capacitación para la inclusión educativa, campañas de sensibilización y exigencias de que se acoja a los estudiantes con NEE y promueve en el discurso de una educación inclusiva, parece ser que en la práctica se constata que los docentes no conocen lo que significa y lo que supone el DUA.

En la escuela pública y en aquellas instituciones donde existe un porcentaje de estudiantes con discapacidad, solo el $29 \%$ sabe lo que significa el DUA, destacando que en la mayoría de los casos son aproximaciones a una definición real del mismo, vinculándolas a la ayuda, a una metodología o bien relacionándola con las dificultades de aprendizaje.

Tabla 1. Presencia de estudiantes con discapacidad en las aulas

\begin{tabular}{|c|c|c|}
\hline Estudiantes con discapacidad en las aulas & Sí & No \\
\hline Pichincha & $29 \%$ & $71 \%$ \\
\hline Sucumbíos & $29 \%$ & $71 \%$ \\
\hline Santo Domingo & $100 \%$ & \\
\hline Loja & $60 \%$ & $40 \%$ \\
\hline
\end{tabular}


Tabla 2. Conocimiento del Diseño Universal para el Aprendizaje

\begin{tabular}{|c|c|c|}
\hline Conocimiento del DUA & Sí & No \\
\hline Pichincha & $14 \%$ & $86 \%$ \\
\hline Sucumbios & & $100 \%$ \\
\hline Santo Domingo & $29 \%$ & $71 \%$ \\
\hline Loja & & $100 \%$ \\
\hline
\end{tabular}

En todos los casos, el profesorado es consciente de la cantidad de información que ellos transmiten, pero que pasa desapercibida para el alumnado, siendo la institución de Sucumbíos la que indica, en un $43 \%$, que la información desapercibida puede llegar alcanzar entre el 60\%$70 \%$ del total de lo que transmiten.

\section{Principio de representación}

Respecto a ítem 5 del cuestionario ¿ofrece alternativas para la información auditiva?, por ejemplo, subtítulos en las presentaciones, uso de diagramas gráficos. Destaca la escuela fiscomisional de Loja al indicar en un $40 \%$ que siempre contempla estas alternativas. Mientras, los docentes de la escuela intercultural bilingüe afirman utilizarlo casi siembre en un 57\%, no encontrando situaciones en la que la respuesta sea nunca.

No ocurre lo mismo cuando hacemos referencia al ítem 6 que responde a si se facilitan alternativas para la información visual, por ejemplo, descripciones texto voz en imágenes. Tal y como se refleja en la tabla 3, el 60\% de los docentes de la escuela fiscomisional indican que utilizan siempre tales alternativas. Por un lado, el $29 \%$ de los docentes de la escuela intercultural bilingüe indican que las utilizan siempre, y el $14 \%$ de los docentes de la misma institución no utiliza nunca esas opciones estratégicas para proporcionar la información a través de formatos alternativos visuales.

Tabla 3. Alternativas para la información visual

\begin{tabular}{|c|c|c|c|c|}
\hline Alternativas visuales & Pichincha & Loja & Santo Domingo & Sucumbíos \\
\hline Siempre & $43 \%$ & $60 \%$ & $29 \%$ & $29 \%$ \\
\hline Casi siempre & $43 \%$ & $40 \%$ & $43 \%$ & $43 \%$ \\
\hline A veces & $14 \%$ & & $29 \%$ & $14 \%$ \\
\hline Nunca & & & & $14 \%$ \\
\hline
\end{tabular}

Los datos con mayor evidencia ante el ítem 7, que plantea si el docente facilita transcripciones escritas de videos o de documentos sonoros, por ejemplo, letras de canciones, intervenciones de personajes, están en los docentes de Sucumbíos. En la tabla 4 se muestra como tan solo el $28 \%$ de estos docentes, manifiesta que siempre o casi siempre utilizan estas estrategias y, al mismo tiempo, llama la atención que un considerado porcentaje de docentes de todas instituciones educativas indica que no considera nunca las trascripciones escritas de videos y documentos sonoros que principalmente se dirigen a los estudiantes que presentan baja audición o visión. 
Tabla 4. Transcripciones escritas de videos o de documentos sonoros

\begin{tabular}{|c|}
\hline Transcripciones escritas \\
\hline Siempre \\
\hline Casi siempre \\
\hline A veces \\
\hline Nunca \\
\hline
\end{tabular}

Ante el ítem 10 ¿Presenta los conceptos clave en formas alternativas al texto?, por ejemplo, mediante imágenes, movimiento, fotografía, material físico o manipulables, y tal y como se muestra en la tabla 5, el mayor porcentaje de docentes que utilizan siempre estas estrategias

\begin{tabular}{|c|c|c|c|}
\hline Pichincha & Loja & Santo Domingo & Sucumbíos \\
\hline $43 \%$ & $40 \%$ & $14 \%$ & $14 \%$ \\
\hline $43 \%$ & & $29 \%$ & $14 \%$ \\
\hline $14 \%$ & $40 \%$ & $43 \%$ & $57 \%$ \\
\hline
\end{tabular}

son los vinculados a la educación fiscomisional de Loja, identificados con el 60\%, a continuación, se encuentran los docentes de la escuela particular e intercultural bilingüe (Sucumbíos), y la particular de Pichincha, ambos con un $43 \%$.

Tabla 5. Presentación de los conceptos clave de forma alternativa al texto

\begin{tabular}{|c|c|c|c|c|}
\hline Presentación alternativa al texto & Pichincha & Loja & Santo Domingo & Sucumbíos \\
\hline Siempre & $29 \%$ & $60 \%$ & $14 \%$ & $29 \%$ \\
\hline Casi siempre & $43 \%$ & $20 \%$ & $14 \%$ & $43 \%$ \\
\hline A veces & $29 \%$ & $20 \%$ & $71 \%$ & $29 \%$ \\
\hline Nunca & & & & \\
\hline
\end{tabular}

\section{Principio de acción y expresión}

Tal y como se puede evidenciar en la tabla 6 , los resultados al ítem 13 ¿Utiliza herramientas tecnológicas de asistencia?, por ejemplo, pantallas táctiles, software accesible, comandos del teclado para el ratón, joysticks, entre el $14 \%$ y el $20 \%$ de los docentes dicen hacer siempre uso de esas estrategias, siendo de mayor uso en la institución fiscomisional, mientras que un porcentaje comprendido entre el $14 \%$ y el $43 \%$ dice que nunca las utiliza. Es la institución fiscal la que revela el mayor porcentaje en el no uso de estas estrategias de asistencia. Resulta revelador este dato, dado que es en las escuelas fiscales en las que se encuentra el mayor número de estudiantes que presentan algún tipo de discapacidad.

Tabla 6. Uso de herramientas tecnologías de asistencia

\begin{tabular}{|c|c|c|c|c|}
\hline Tecnología de asistencia & Pichincha & Loja & Santo Domingo & Sucumbíos \\
\hline Siempre & $14 \%$ & $20 \%$ & $14 \%$ & $14 \%$ \\
\hline Casi siempre & $14 \%$ & $20 \%$ & & $14 \%$ \\
\hline A veces & $43 \%$ & $60 \%$ & $43 \%$ & $57 \%$ \\
\hline Nunca & $29 \%$ & & $43 \%$ & $14 \%$ \\
\hline
\end{tabular}




\section{Múltiples medios de implicación}

En cuanto al ítem 20, ¿El desarrollo de contenidos considera el interés de los estudiantes de acuerdo con la diversidad?, por ejemplo, culturas, etnias, condición de discapacidad, los resultados obtenidos reflejan que la institución fiscomisional manifiesta que un $60 \%$ siempre lo hace. A continuación, se encuentra la institución intercultural bilingüe, en la que un 57\% del profesorado indica la opción casi siempre, frente a un $14 \%$ de la escuela fiscal que manifiesta no realizarlo nunca. De modo que existe un porcentaje importante del profesorado que no reconoce el interés de cada estudiante $y$, por tanto, no personaliza la enseñanza.

Atendiendo al ítem 26 ¿Organiza comunidades o grupos de aprendizaje centrados en intereses o actividades comunes de los estudiantes?, tal y como se desprende de los datos analizados, la escuela intercultural bilingüe marca la opción siempre representada con el $43 \%$, seguida de los docentes de la institución fiscomisional representada con el 40\%. Las intuiciones educativas particular y fiscal, marcan la opción nunca, representada por el $14 \%$.

Por último, el ítem 29 plantea la cuestión ¿Favorece el reconocimiento de los propios progresos en los estudiantes? se observa cómo los docentes de la escuela fiscomisional indican la opción siempre con el $80 \%$ de respuestas, seguido de los docentes de la escuela particular e intercultural bilingüe con la opción casi siempre, representada el 57\%. Resulta trascendente señalar que ningún docente marcó la opción nunca, lo que deja comprender que en todas las instituciones existe la práctica de fomentar el reconocimiento de los propios progresos, asegurando que el estudiante sea consciente de su propio proceso de aprendizaje. No obstante, es preocupante el hecho de que esto no sea una práctica habitual al $100 \%$ en todos los centros.

Respecto a las preguntas abiertas, ante la pregunta ¿qué entiende por educación inclusiva?, los docentes de la escuela fiscal (Santo Domingo) inciden en sus definiciones en aspectos relacionados con el acceso a la educación, la diversidad en las capacidades, en modificaciones de la metodología, junto con la igualdad de oportunidades. Por otro lado, la escuela intercultural bilingüe (Sucumbíos) ponen el acento en el aprender juntos, la formación continua y la igualdad de oportunidades. Los docentes de la escuela fisco misional (Loja), se decantan más hacia clases que acogen a la diversidad, la inclusión en el entorno, los cambios metodológicos y la ayuda. Por último, desde la escuela particular (Quito), hacer referencia al derecho a la educación, a la multitud de diversidad y a los cambios metodológicos. Respecto a las actividades exitosas para favorecer la inclusión, destacan en prácticamente la totalidad de los casos actividades de sensibilización utilizando juegos y cuentos. Y por ultimo, todos coinciden en la necesidad de formación en DUA.

\section{Discusión y conclusiones}

De acuerdo con los objetivos planteados al inicio de nuestra investigación, ha resultado evidente que existe un conocimiento limitado sobre el DUA, y, por ende, de la aplicación de las estrategias metodológicas relacionadas con el mismo. Sin embargo, los resultados también aportan información sobre el uso de estrategias didácticas utilizadas por los docentes de diferentes áreas geográficas que invitan a la reflexión sobre el proceso de implantación de la educación inclusiva y de las diferentes actitudes o percepciones sobre los tipos de diversidad. A la vista de los datos obtenidos para cada uno de los principios del diseño universal para el aprendizaje, resulta preocupante la ausencia de las alternativas a la información visual, ya que este aspecto refleja que un alto número de estudiantes no dispone de adecuada accesibilidad a la información, afectando principalmente al alumnado que presenta baja visión. Como afirma Alba (2015), "para superar esta barrera hay que proporcionar la información a 
través de otros formatos alternativos, no visuales" (Alba, Sánchez, \& Zubillaga, 2013).

Por otro lado, nos hace reflexionar el alto porcentaje de profesorado que no utiliza transcripciones escritas de videos o de documentos sonoros en todas las instituciones educativas. Este hecho puede suponer que esté afectando tanto al proceso formativo como a la igualdad de oportunidades del estudiantado que presenta baja audición o visión. Según Alba (2015), las transcripciones proporcionan opciones que ayudan a reducir las barreras o dificultades relacionadas con la decodificación de símbolos que no resultan familiares. De este modo, se considera necesario fomentar el uso de alternativas de texto que permitan al alumno comprender significados complejos.

Sin embargo, es importante destacar que, en lo relativo a la presentación de conceptos clave de forma alternativa al texto (como puede ser a través de la utilización de imágenes, videos, fotografías) sí que es tenido en cuenta por el profesorado. Esto puede deberse a que al ser conceptos clave son momentos puntuales en el aula y no requieren una gran inversión de tiempo para su elaboración. Contar con un trabajo colaborativo con el conjunto del profesorado, dirigido a la planificación de contenidos basados en diseño universal para el aprendizaje reduciría el tiempo invertido en el diseño de materiales y, por tanto, fomentaría y permitía una mayor comprensión de los contenidos.

Por otro lado, este tipo de coordinación docente supondría de hecho desarrollar la capacidad creadora del profesorado ya que de manera conjunta desarrollaría materiales y/o estrategias para poder llegar con la información a todos los estudiantes, tomando en cuenta las diferencias individuales y los diferentes tipos de inteligencias existentes en el aula de clase. Respecto al principio de acción y expresión destaca la escasez de herramientas tecnológicas de asistencia, como sillas de ruedas, prótesis, comunicadores y servicios de telecomunicaciones (Sánchez, 2018). Al respecto, se puede evidenciar que la institución que no revela ningún porcentaje en el uso de estas estrategias de asistencia es la escuela fiscal. Cabe recordar que la escuela fiscal obtuvo mayor porcentaje en cuanto a la presencia de estudiantes con discapacidad. Resulta preocupante que sean los centros que cuentan en sus aulas con un mayor numero de estudiantes con discapacidad aquellos que no hacen uso de tecnologías asistenciales, aun cuando estas son consideradas como un pilar fundamental para generar comunidades inclusivas de aprendizaje. Esto puede deberse a que la tecnología de asistencia requiere de un financiamiento que puede ser representativo para la escuela.

Respecto a los medios de implicación, debemos considerar que, aunque en general si se tienen en cuenta, el hecho de que exista un alto porcentaje de docentes que contempla los intereses del alumnado debe ser un aspecto por mejorar, ya que la mayoría de las veces estos intereses están vinculados a su identidad cultural y social, lo cual ayuda a que la educación sea más personalizada brindando aprendizajes significativos al estudiante. Este aprendizaje se denomina "aprendizaje significativo" y ocurre cuando una nueva información se conecta con un concepto relevante, preexistente en la estructura cognitiva (Chrobak, 2017).

Por otro lado, el DUA plantea la realización de actividades comunes a través de la creación de comunidades de aprendizaje, debiendo ser estas a su vez comunes en intereses. $\mathrm{Al}$ respecto, la escuela intercultural bilingüe, seguida de los docentes de la institución fiscomisional, representa las acciones más continuadas. El hecho de que las intuiciones educativas particular y fiscal marquen la opción "nunca", se puede atribuir a que, en la gran ciudad, "contradictoriamente", existe facilidad de comunicación y al tratarse de niños, no es posible el encuentro para el aprendizaje en comunidad, lo que sí es más viable en las poblaciones pequeñas. El interés que prestan los docentes de la escuela intercultural bilingüe por formar comunidades de aprendizaje puede deberse al factor cultural, pero al mismo tiempo llama la atención que el resultado no haya sido el $100 \%$ considerando que los docentes son 
profesionales nativos de la zona. Este principio refleja una concepción histórica cultural del desarrollo, lo cual manifiesta que, el conocimiento no se construye de modo individual, sino que se construye entre las personas a medida que interactúan (Duque, \& Packer, 2014).Otro de los intereses del DUA es favorecer el reconocimiento de los propios progresos en los estudiantes para mejorar la calidad educativa y, al respecto, resulta trascendente señalar que ningún docente marcó la opción "nunca", lo que deja comprender que en todas las instituciones existe la práctica de fomentar el reconocimiento de los propios progresos, asegurando que el estudiante sea consciente de su propio proceso de aprendizaje.

No obstante, es preocupante el hecho de que esto no sea una práctica habitual al $100 \%$ en todos los centros. A través de la retroalimentación el estudiante dispone de mas información, y, por tanto, le permite aproximarse a la situación ideal a la que debe llegar. Es decir, pretende eliminar la distancia entre el desempeño actual y el que pretende alcanzar (Ramaprasad,1983; Roos, 2004). Por lo que se puede afirmar, en relación con nuestro objetivo, que existe un conocimiento muy limitado acerca del DUA, y, por ende, la aplicación de las estrategias metodológicas relacionadas con los principios de este es aún más escasas.

En lo referente al objetivo de conocer sí existe relación entre la ubicación geográfica e inclusión educativa y el conocimiento y uso del DUA, resulta evidente que los docentes que se encuentran a mayor distancia de las ciudades tienen menos oportunidades para generar un proceso de educación en igualdad de oportunidades garantizando una educación de calidad como lo proclama el DUA. Sin embargo, es necesario destacar que en lo que respecta a indicadores relacionados con el principio de múltiples medios de implicación, más vinculados con aspectos motivacionales y emocionales, las instituciones fronterizas con otros países y/o aquellas que disfrutan de una identidad cultural acentuada y arraigada parece que tienen más interiorizados sistemas de funcionamiento vinculados a la comunidad y a su relación con esta, percibiéndose similitudes con metodologías didácticas activas como podría ser el aprendizaje cooperativo, que presenta unas características inmejorables para el fomento de la inclusión educativa.

Por tanto, podemos pensar que no todas las estrategias metodológicas tradicionales ralentizan el proceso de inclusión educativa; tal es el caso de aquellas que vienen de pensamientos ancestrales que toman en cuenta la supervivencia como grupo étnico o grupo lingüístico, como la práctica de narraciones que utilizan los docentes en la escuela intercultural bilingüe y de los encuentros formativos alrededor de la "toma de guayusa".

De este modo, coincidimos con Martínez (2014), al hacer referencia a la construcción del aprendizaje a partir del análisis las experiencias vividas y que resulten, por tanto, familiares los conceptos e ideas planteadas, ya que les permitirán alcanzar la resolución de futuros problemas. En cualquier caso, la diversidad es diversa en sí misma y ante esta realidad, aceptar y respetar otras situaciones de diversidad, más allá de la propia, es imprescindible para conseguir una inclusión real.

De ahí que muchos estudiantes experimenten dificultades al no tener en cuenta la diversidad de los procesos de enseñanza y aprendizaje (Booth, \& Ainscow, 2015). En determinadas situaciones se atiende a la diversidad cultural o étnica, pero no se tienen en cuenta otras situaciones como la diversidad en el funcionamiento de las personas, y esto solo puede suponer la exclusión de estas. Los diversos grupos sociales, etnias y culturas tienen normas, valores, creencias y comportamientos distintos, que generalmente no forman parte de la cultura escolar, lo que puede limitar sus posibilidades de aprendizaje y de participación, o conducir a la exclusión y discriminación (Echeita, 2018).

A la vista de los resultados analizados junto con las propias demandas de los docentes, podemos concluir con la necesidad de seguir proporcionando formación a los docentes relacionada con la calidad de la educación, la inclusión edu- 
cativa y la igualdad de oportunidades, ya que las acciones realizadas hasta el momento parecen no ser suficientes. Por lo tanto, la formación docente con relación a la atención a la diversidad e inclusión educativa y al DUA se vuelve una necesidad urgente para brindar a los docentes herramientas ya no solo para pensar en la educación como un derecho de todos, sino para llevar a la práctica tal enfoque, pues, nada más dinámico y desafiante para el docente es el desarrollo integral de todos los niños. En cualquier caso, consideramos necesaria la participación de las universidades en la formación en estas áreas de conocimiento tanto en la formación de base de los docentes como en formación especializada como las maestrías, junto con la revisión, ampliación y creación de planes y/o proyectos que incentiven las políticas públicas de Ecuador en la capacitación de los docentes en general y de educación básica en particular. La atención a la diversidad es uno de los requerimientos que la actual legislación hace a las instituciones educativas; por tanto, "la necesidad de formar a los profesores en la aplicación del paradigma del diseño universal para el aprendizaje y sus ventajas a la hora de proporcionar las mismas oportunidades para todos los estudiantes, independientemente de sus características individuales, es sin duda una forma para mejorar la atención a la diversidad" (Diez \& Sánchez, 2015).

Ante esto se puede decir que quien tiene acceso a la comunicación adquiere la información y, por lo tanto, accede al conocimiento. Si los docentes no tienen acceso a la información, los procesos educativos con vistas a brindar educación en igualdad de oportunidades se tornan más lentos que en los espacios donde hay más información y los medios la favorecen.

A partir de la expedición en el año 2008 de la nueva Constitución de la República se estable que "la educación es un derecho de las personas a lo largo de su vida y un deber ineludible e inexcusable del Estado. Constituye un área prioritaria de la política pública y de la inversión estatal, garantía de la igualdad e inclusión social y con- dición indispensable para el buen vivir (art. 26). Además, en el artículo 27 de la misma normativa se establece que la educación debe además de ser "participativa, obligatoria, intercultural, democrática, incluyente y diversa, de calidad y calidez"; es decir, que todas las personas deben ser incluidas en el sistema educativo (Ministerio de Educación, 2012).

Al finalizar la investigación, se puede decir que el DUA se coloca como una alternativa didáctica en el marco de la educación inclusiva. Responde a la necesidad de romper con el enfoque homogeneizante de la educación tradicional, que considera que todos los estudiantes aprenden de la misma forma y que el rol docente es el de organizar y preparar los materiales con el mismo formato para todos, fomentado la exclusión antes que la inclusión. En cualquier caso, este estudio nos describe y acerca a una realidad, pero para poder tener más evidencias de la situación sería necesario realizar un estudio a nivel nacional, que contara con un mayor número de población muestral, ya que, aunque relevante, este hecho supone una limitación para generalizar o realizar afirmaciones absolutas.

\section{Referencias bibliográficas}

Alba, C. (2015). Aportaciones del Diseño Universal para el Aprendizaje y de los materiales digitales para el logro de una enseñanza accesible. En J. Navarro, M. Fernández, F. Soto \& F. Tortosa, (Ed.), Respuestas flexibles en contextos educativos diversos, $1^{\circ}$ ed. Murcia: Región de Murcia Consejería de Educación (https://bit.ly/2ccLNvf) (2019//01/23).

Alba, C., Sánchez, P., \& Zubillaga, A. (2013). Pautas sobre el Diseño Universal de Aprendizaje 2.0 (versión traducida al español). Madrid. (https://bit.ly/2Wnczbf) (2019/01/22)

Asamblea Constituyente (2008). Constitución de la República del Ecuador. Quito.

Ausubel, D. P. (2002). Adquisición y retención del conocimiento. Una perspectiva cognitiva. Barcelona: Ed. Paidós.

Barrera, L. (2009). Neurociencias y su importancia en contextos de aprendizaje. 
Revista Digital Universitaria, 10(4), 1-18. (https://bit.ly/2I54T60) (2019/01/25).

Benítez, L., \& Garcés, A. (2016). Culturas ayer y hoy. Quito: Abya-Yala.

Blanco, R., \& Duck, C. (2011). Educación Inclusiva en América Latina y el Caribe. Aula, (17), 37-55. (https://bit.ly/2B9d) (2019/01/29).

Booth, T., \& Ainscow, M. (2015). Guía para la educación inclusiva. Madrid.

(https://bit.ly/2EhiLu4) (2019/01/28/)

CAST (2011). Universal Design for Learning Guidelines versión 2.0. Wakefield, MA: Author. Traducción al español versión 2.0. (2013). (https://bit.ly/2IaxAP6) (2019/01/27).

Chrobak, R. (2017). El aprendizaje significativo para fomentar el pensamiento crítico. Archivos de ciencia de la Educación, 11(12), 1-12. https://doi.org/10.24215/23468866e 031

Díez, E., \& Sánchez, S. (2015). Diseño Universal para el aprendizaje como metodología docente para atender a la diversidad en la universidad. Aula Abierta, (43), 87-93. (https://bit.ly/2HD8w7n) (2019/01/29).

Duque, M., \& Packer, M. (2014). Pensamiento y lenguaje. El proyecto de Vygotsky para resolver la crisis de la piscología. Tesis Piscología, 9(2), 30-57. (https://bit.ly/2WrgJ1F) (2019/01/20).

Echeita, G. (2018). Educación para la inclusión o educación sin exclusiones. Madrid: Narcea.

Espada, R. (2017). Bases psicopedagógicas y detección de necesidades educativas especiales. En Máster en Atención a Necesidades Educativas Especiales para Niveles de Educación Infantil $y$ Primaria. Universidad Rey Juan Carlos. (https://bit.ly/2MJGqpX) (2018/12/21).
Martínez, J. (2014). El sueño oriental. O la dificultad de la incorporación del territorio amazónico al estado nacional en Ecuador. Antropología, (14), 37-64. (https://bit.ly/2DGwqup) (2019/01/10) .

Ministerio de Educación (2011). Ley Orgánica de Educación Intercultural. Quito: Ministerio de Educación.

Quecedo, R., \& Castaño, C. (2003). Introducción a la metodología de investigación cualitativa. Revista de Psicodidáctica, 14, 5-40.

Ramaprasad, A. (1983). On the definition of feedback. Behavioral Science, 28, 4-13. (https://bit.ly/2IYqnFI) (2019/01/10).

Roos, B. (2004). Learning and Assessment in the Knowledge Society. Umea: Universidad de Umea, en EDEN 2004 Annual Conference, New challenges and Partnerships in an enlarged European Union: Open, Distance and e-Learning in Support of Modernisation, Capacity Building and Regional Development, 16-19 June, University of Technology and Economics, Budapest, Hungary.

Sánchez, R. (2018). La inclusión de las TIC en la educación de las personas con discapacidad. Quito: Abya-Yala.

Sánchez, S., Díez, E., \& Martín, R. (2016). El Diseño Universal para atender a la diversidad en la educación. Contextos Educativos, 9, 121-131. https://doi.org/10.18172/con.2752

UNESCO (1994). Declaración de Salamanca. Salamanca: UNESCO. (https://bit. ly/1wiQz1c) (2019/01/15).

UNESCO (2015). Declaración de Incheon y Marco de Acción para la realización del Objetivo de Desarrollo Sostenible 4. Francia: UNESCO. (https://bit.ly/2ejZBWa) (2019/01/15). 